\title{
Comparaison de la sensibilité du test de Woo et d'un test de détection des antigènes de Trypanosoma vivax chez deux moutons expérimentalement infectés avec une souche guyanaise du parasite
}

\author{
M. Desquesnes ${ }^{1}$, S. de La Rocque ${ }^{1 *}$
}

DESQUESNES (M.), LA ROCQUE (S. de). Comparaison de la sensibilité du test de Woo et d'un test de détection des antigènes de Trypanosoma vivax chez deux moutons expérimentalement infectés avec une souche guyanaise du parasite. Revue Élev. Méd. vét. Pays trop., 1995, 48 (3) : 247-253

Un ELISA de détection des antigènes circulants de Trypanosoma vivax, par immunocapture à l'aide d'un anticorps monoclonal spécifique, a été mis au point par Nantulya et Lindqvist en 1989 : sur les souches africaines testées sa sensibilité s'est avérée très élevée. Avec des réactifs fournis par l'IRLI, la sensibilité de cette méthode a été comparée à celle du test de Woo lors d'infections expérimentales de deux moutons avec une souche guyanaise de $T$, vivax. Des tests, réalisés en aveugle au CIRDES et à l'ILRI, ont permis de confirmer les résultats obtenus en Guyane française. Les animaux ont été régulièrement prélevés pendant les 130 et 285 premiers jours d'infection. Quelle que soit la période d'infection, que la parasitémie fût détectable ou non, la sensibilité du test ELISA a été très basse : en moyenne 2,1 p. 100 de résultats positifs, de loin inférieure à celle de la technique de Woo : 54 p. 100 de résultats positifs. L'association des deux techniques n'apporte aucune sensibilité supplémentaire. Les densités optiques moyennes enregistrées au cours de ces longues périodes d'infections, $0,010 \pm 0,004$ et $0,012 \pm 0,002$, sont si proches du seuil de sensibilité des lecteurs ELISA qu'elles ne peuvent être proposées comme seuil de positivité du test, pour en améliorer la sensibilité. Une étude complémentaire de la sensibilité de ce test est en cours à I'ILRI. Déjà observé au Burkina Faso et en Gambie, ce défaut de sensibilité risque d'affecter fortement les résultats des enquêtes épidémiologiques entreprises avec ces réactifs en Afrique. Il est nécessaire de développer de nouveaux anticorps monoclonaux afin de mettre au point un test de détection des antigènes de $T$. vivax dont la sensibilité soit plus satisfaisante.

Mots-clés : Ovin - Trypanosoma vivax - Infection expérimentale - Test ELISA - Antigène - Anticorps - Sérologie - Guyane française.

\section{INTRODUCTION}

Pour la détection des infections du bétail par Trypanosoma vivax, le diagnostic parasitologique est peu sensible car les parasitémies sont souvent trop basses. L'observation de l'interface sang/plasma en tube capillaire, ou test de Woo (14), est souvent utilisée comme référence pour évaluer la sensibilité de nouvelles techniques de diagnostic des infections actives par les trypanosomes.

1. CIRAD-EMVT-Guyane, Institut Pasteur, BP 6010, 97306 Cayenne, Guyane, Francc.

* Adresse actuelle : CIRDES, 01 BP 454, Bobo-Dioulasso 01, Burkina Faso.

Reçu le 2.11.1993, accepté le 5.12.1995.
Un ELISA pour la détection des antigènes circulants de Trypanosoma vivax par immunocapture a été mis au point à partir d'une souche africaine du parasite, par Nantulya $(9,10)$, Nantulya et Lindqvist (11), Nantulya et al. (12) à l'ILRI* (anciennement ILRAD ${ }^{* *}$ ). Ce test est actuellement en cours d'évaluation par la division conjointe FAO/IAEA à Vienne. Les réactifs utilisés dans cette expérimentation ont été fournis gracieusement par I'ILRI au CIRDES ${ }^{\star \star \star}$ (anciennement CRTA ${ }^{\star \star \star \star}$ ) et au CIRAD-EMVT en Guyane française. Une souche de Trypanosoma vivax a été isolée en Guyane française, par Lancelot en 1988 (7).

Dans la présente étude, pour comparer la sensibilité du test de Woo et de cet ELISA, la souche guyanaise a été inoculée expérimentalement à 2 moutons, régulièrement prélevés pendant 4,5 et 9,5 mois. Les tests parasitologiques et sérologiques ont été réalisés au laboratoire du CIRAD-EMVT en Guyane française. Pour confirmer ces résultats, un échantillonnage de sérums a également été transporté à l'ILRI et au CIRDES afin d'effectuer une partie des tests en aveugle.

\section{MATÉRIEL et MÉTHODES}

\section{Matériel}

\section{Animaux}

Deux animaux âgés de 2 ans, un mâle (mouton 1) et une femelle gestante (mouton 2), issus de croisements Blackbelly $\times$ Créole, ont été placés dans des bergeries protégées par des moustiquaires afin d'éviter toute dissémination des parasites et toute contamination exogène. Les animaux sont nourris à base de foin (Brachiaria decumbens) et de complément alimentaire commercial.

Pour s'assurer que les animaux n'étaient pas infectés au préalable, les tests suivants ont été réalisés : test de Woo (14) et Murray et al. (8), examens de frottis san-

\footnotetext{
* International Livestock Research Institute, Nairobi, Kenya.

** International Laboratory for Research on Animal Diseases, Nairobi, Kenya.

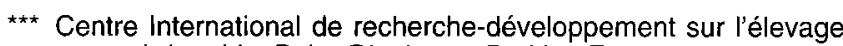
en zone sub-humide, Bobo-Dioulasso, Burkina Faso.

**** Centre de recherche sur les trypanosomes animales, BoboDioulasso, Burkina Faso.
}

On citera tout au long de l'article les noms des nouveaux centres (ILRI pour ILRAD, CIRDES pour CRTA). 
guins colorés, détection des antigènes de Trypanosoma vivax, $T$. brucei et $T$. congolense par ELISA (11) et détection des anticorps dirigés contre Trypanosoma spp. par la méthode ELISA de Ferenc et al. (4).

\section{Souche de T. vivax Guyane}

La souche de T. vivax utilisée a été isolée à partir d'un zébu, cultivée sur veau Frison (un passage) et sur mouton Créole (un passage), avant d'être stockée en azote liquide (7). Un stabilat de 1,5 ml, préparé en 1989, a été inoculé au mouton 1. II était pauvre en parasites, environ $10^{3}$ par $\mathrm{ml}$. Lors d'un pic parasitémique chez cet animal, du sang frais parasité a été utilisé pour infecter le mouton 2 .

\section{ELISA par immunocapture}

Le test de détection des antigènes de $T$. vivax repose sur un anticorps monoclonal obtenu à partir de formes procycliques d'un parasite africain (12). L'antigène reconnu présentant plusieurs épitopes identiques, l'anticorps monoclonal est utilisé à la fois pour la sensibilisation de la plaque et pour la révélation des antigènes (conjugaison du monoclonal à une peroxydase).

Dans cette expérience, deux types de plaques ELISA sont utilisées. Les tests sont réalisés en Guyane française avec les plaques de microtitration MAXISORP NUNC ${ }^{\mathrm{ND}}$, tandis que des plaques IMMULON B DYNA$\mathrm{TECH}^{\mathrm{ND}}$ sont utilisées au CIRDES et à ILRI. La comparaison ayant été faite antérieurement, il n'est pas attendu de différence importante entre ces deux types de plaques (G. Duvallet, communication personnelle).

\section{Méthode}

\section{Infections expérimentales}

Le stabilat est ramené à température ambiante par un bref séjour à $37^{\circ} \mathrm{C}$, il est vérifié que les parasites sont vivants avant inoculation. L'inoculation des parasites a été faite à la veine jugulaire des moutons à J0 :

- environ 1500 parasites pour le mouton 1 ;

- environ $1,5 \times 10^{6}$ pour le mouton 2 .

\section{Traitements}

Les animaux ont été traités à l'acéturate de diminazène (Berenil ${ }^{\mathrm{ND}}$ ) à la dose de $3,5 \mathrm{mg} / \mathrm{kg}$ par voie intra-musculaire (IM) lorsque la parasitémie dépassait $10^{7} / \mathrm{ml}$, afin de pouvoir évaluer la persistance des antigènes dans la circulation sanguine après la mort des parasites, comme indiqué par Nantulya et Lindqvist (11). Avec cette souche de parasites, on savait qu'à la dose de $3,5 \mathrm{mg} / \mathrm{kg}$ d'acéturate de diminazène la chute brutale de la parasitémie serait réversible, et qu'en conséquence, l'infection persisterait (2).

Les traitements successifs à l'acéturate de diminazène favorisant le contrôle de la parasitémie, on a tenté d'induire une période aparasitémique en traitant à nouveau le mouton 1 au $130^{\circ}$ jour d'infection, bien que la parasitémie n'atteignît pas $10^{7}$.

\section{Collecte des échantillons}

Dans la mesure du possible, les animaux sont prélevés quotidiennement. Le sang est collecté sur vacutainers héparinés pour les examens parasitologiques, sur tubes secs pour la sérologie. Les examens parasitologiques sont effectués dans les minutes qui suivent la collecte. Le sérum est récolté 6 heures après le prélèvement (centrifugation $430 \mathrm{~g}$ pendant 10 minutes), puis conservé à $-20^{\circ} \mathrm{C}$, jusqu'à la réalisation des tests ELISA.

\section{Analyses parasitologiques}

Le test de Woo, le comptage sur cellule et l'examen de frottis ont été réalisés.

L'interface de tubes à hématocrite est examiné après centrifugation de $14000 \mathrm{~g}$ pendant cinq minutes, comme décrit par Woo (14). La sensibilité du test est de 'l'ordre de $10^{3}$ trypanosomes/ml $(1,8,14)$.

Le comptage est réalisé en cellule de Thoma, après dilution du sang, au 1/10 ou au 1/100 (selon la parasitémie), dans une solution tamponnée de phosphate, salée et glucosée (PSG, pH 7,4) ; il permet une estimation de la parasitémie.

Les frottis colorés sont examinés pour vérifier l'absence d'autres parasites et confirmer la présence de $T$. vivax sur les critères de taille et de morphologie indiqués par Hoare (5).

\section{Sérodiagnostic par ELISA}

La méthode utilisée est décrite par Nantulya et Lindqvist (11). Le résultat est considéré comme positif si la densité optique de l'échantillon est' supérieure à 0,05 . Pour faciliter la lecture des résultats, les densités optiques indiquées par le lecteur ELISA sont multipliées par 1000 , et l'on note DO cette valeur. Des sérums témoins, 2 positifs et 2 négatifs, fournis par l'ILRI, sont disposés sur chaque plaque de microtitration, afin de vérifier que leurs valeurs ne s'éloignent pas de celles usuellement obtenues. Dans le cas contraire, l'ensemble des résultats fournis par cette plaque serait rejeté. Les comparaisons de moyennes sont réalisées entre les moyennes des DO des animaux infectés et celles des témoins, par le test $d u$ " $t$ " décrit par Schwartz (13). 


\section{Test en aveugle}

Afin d'être testés à l'ILRI et au CIRDES, 42 échantillons présentant, en Guyane, un large panel de densités optiques et de parasitémies ont été sélectionnés parmi les 330 prélèvements réalisés au cours de l'expérience. Les résultats des trois laboratoires seront comparés. Des aliquotes de ces sérums ont été transportées sous froid (carboglace) jusqu'aux laboratoires en Afrique. L'analyse statistique permettra d'établir si les résultats des trois laboratoires sont significativement différents, ou non; elle est réalisée selon les méthodes décrites par Schwartz (13):

- analyse qualitative de séries appariées (test du CHI 2) ;

- analyse quantitative de séries appariées (comparaison de moyennes des DO).

\section{RÉSULTATS}

Avant l'inoculation, toutes les techniques de détection de Trypanosoma sp. étaient négatives, révélant que ces animaux n'ont pas eu de contact préalable avec des trypanosomes pathogènes.

\section{Résultats parasitologiques}

Pour des raisons pratiques, le mouton 1 n'a pu être prélevé de J165 à J190 et de J200 à J225. En dehors de ces périodes, les prélèvements n'ont jamais été espacés de plus de trois jours.

\section{Mouton 1 (mâle)}

Sur 285 jours de suivi l'animal a été prélevé 205 fois ; le test de Woo a été positif 90 fois (44 p. 100). Le test de Woo est devenu positif à J4. La figure 1 montre l'évolution de la parasitémie qui atteint $1,4 \times 10^{7}$ trypanosomes $/ \mathrm{ml}$ à J12. A ce stade, un traitement à l'acéturate de diminazène à la dose de $3,5 \mathrm{mg} / \mathrm{kg}$ a été réalisé. La parasitémie s'est annulée le lendemain du traitement, mais les trypanosomes ont à nouveau été détectés une dizaine de jours plus tard. La parasitémie a ensuite été élevée, justifiant un traitement à J36 (parasitémie $>10^{7}$ ). De la même manière, les trypanosomes ont disparu puis ont à nouveau été détectés dans le sang circulant 9 jours après le traitement (J45). Par la suite, la parasitémie est restée inférieure au plafond fixé pour le traitement $\left(10^{7}\right)$; l'animal a toutefois été traité à J130 pour favoriser l'apparition d'une période aparasitémique. Les examens parasitologiques sont alors restés négatifs pendant plus de 4 mois.

A l'occasion d'un stress alimentaire (rupture d'approvisionnement en granulés pendant deux jours), les parasites ont été détectés dans le sang de manière très fuga-
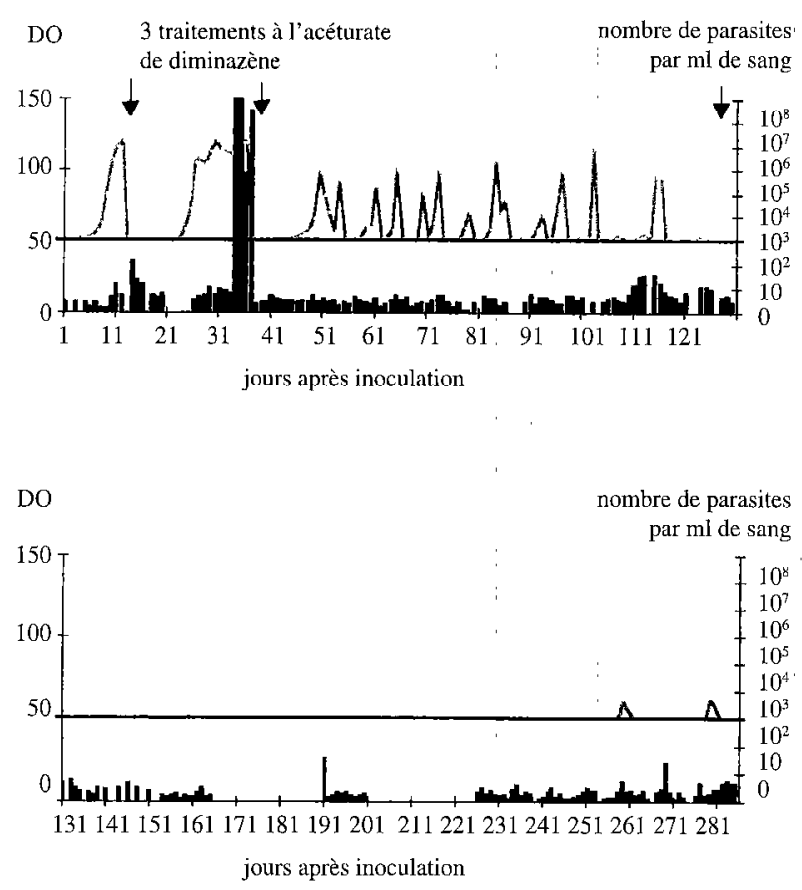

Figure 1 : Parasitémie, traitements et DO fournies par les tests ELISA du mouton 1 (haut : période 1, J1-J130; bas : période 2, J131-J285).

Histogrammes: valeurs des DO obtenues au test de détection des antigènes trapping-ELISA; courbes grisées: parasitémie (échelle logarithmique); barre horizontale: seuil de positivité des deux tests pratiqués (DO 50 pour le test ELISA, et parasitémie $10^{3}$ trypanosomes $/ \mathrm{ml}$ pour le test de Woo); les flèches verticales indiquent les jours de traitement à l'acéturate de diminazène.

ce et peu intense (parasitémies $<104 / \mathrm{ml}$ ), à J259-260, puis, en l'absence de stress alimentaire, à J281-282.

L'évolution de la parasitémie a donc permis de distinguer successivement une période de forte parasitémie ( $\mathrm{J} 1$ $\mathrm{J} 130$ ) et une période de parasitémie quasiment non détectable par le test de Woo (J131-J285) :

- J1-J130 : sur 130 jours de suivi, l'animal a été prélevé 113 fois, le test de Woo a été positif 85 fois (75 p. 100). Les parasitémies, généralement élevées, ont été annulées pendant des périodes de 9 à 10 jours par l'administration d'acéturate de diminazène, ou, naturellement, pendant des périodes de durées inférieures à six jours (fig. 1 : haut) ;

- J131-J285 : sur 155 jours de suivi, l'animal a été prélevé 92 fois, le test de Woo a été positif 5 fois $(5,4$ p. 100). Les parasitémies étaient généralement non décelables ou seulement de manière épisodique; l'animal est toutefois resté porteur du parasite pendant toute la durée du suivi (fig. 1 : bas).

La sensibilité de la technique parasitologique est élevée au début de l'infection. Par la suite, alors que l'animal s'immunise, les parasitémies sont de plus en plus basses. jusqu'à devenir non détectables. La récurrence des para- 
sites dans le sang témoigne cependant de l'infection continue de l'animal pendant toute la période d'observation.

\section{Mouton 2}

Sur 130 jours de suivi, l'animal a été prélevé 125 fois, le test de Woo a été positif 89 fois $(71$ p. 100). Le test de Woo était positif dès le lendemain de l'inoculation; puis la parasitémie a été généralement élevée pendant toute la durée du suivi, atteignant deux fois le seuil fixé pour le traitement (fig. 2). Après les traitements à l'acéturate de diminazène $(3,5 \mathrm{mg} / \mathrm{kg} \mathrm{IM})$, les parasites n'étaient plus détectables pendant 9 et 13 jours.

En moyenne les parasitémies de cet animal ont été plus élevées que celles du mouton 1 pendant les 130 premiers jours de l'infection, dépassant souvent $10^{6}$ parasites/ml de sang; ce phénomène peut être mis en relation avec l'état physiologique de l'animal pendant l'infection (gestation, mise bas à $\mathrm{J} 28$, puis lactation pendant un mois).

\section{Résultats sérologiques}

Les résultats des tests ELISA sont indiqués aux figures 1 et 2 où les histogrammes représentent les DO obtenues aux tests $(D O=1000 \times$ densité optique donnée par le lecteur ELISA). Les échelles des DO et de la parasitémie ont été choisies de telles sorte que le seuil de sensibilité de chaque test apparaît à la même hauteur sur la figure (barre horizontale correspondant à 1000 trypanosomes $/ \mathrm{ml}$ pour le test de Woo et à la DO 50 pour les tests ELISA).

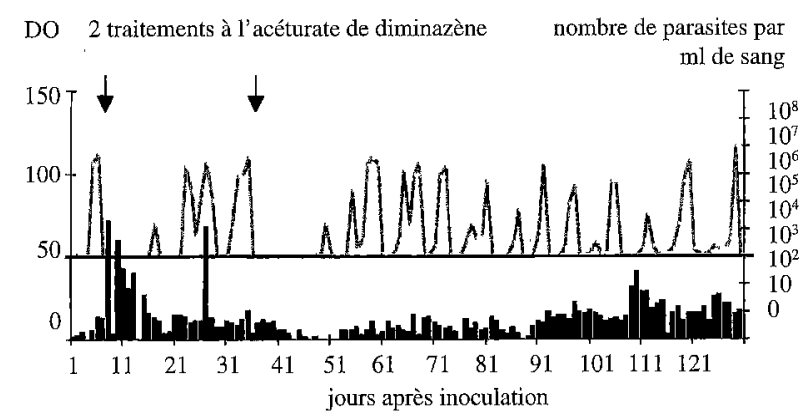

Figure 2 : Parasitémie, traitements et DO fournies par les tests ELISA du mouton 2 .

Histogrammes : valeurs des DO obtenues au test de détection des antigènes trapping-ELISA; courbes grisées: parasitémie (échelle logarithmique); barre horizontale: seuil de positivité des deux tests pratiqués (DO 50 pour le test ELISA, et parasitémic $10^{3}$ trypanosomes/ml pour le test de Woo); les flèches verticales indiquent les jours de traitement à l'acéturate de diminazène.

\section{Mouton 1, période 1 (J1-J130)}

Sur 113 prélèvements, l'ELISA a été positif 4 fois $(3,5 \mathrm{p}$. 100). La DO moyenne sur cette période est de $15 \pm 6$ (intervalle de confiance de la moyenne au risque de $5 \mathrm{p}$. 100). Le test a été positif de J34 à J37. La DO maximale a été de 297.

\section{Mouton 1, période 2 (J131-J285)}

Les 92 prélèvements ont fourni des résultats négatifs. La $\mathrm{DO}$ moyenne sur cette période est de $5 \pm 1$. La DO maximale a été de 25.

Pour ces périodes cumulées, le mouton 1 a été prélevé 205 fois, l'ELISA a été positif 4 fois, la DO moyenne est de $10,5 \pm 4$. Le pourcentage de résultats positifs enregistrés sur la totalité du suivi de cet animal est de 1,9 p. 100.

\section{Mouton 2 (J1-J130)}

Sur 125 prélèvements l'ELISA a été positif 3 fois $(2,4 \mathrm{p}$. 100). La DO moyenne de toute la période d'infection est de $12 \pm 2$. Le test a été positif à J8, J12 et J27. La DO maximale a été de 71 . Les 2 témoins négatifs et les 2 témoins positifs ont été testés 8 fois chacun et ont donné en moyenne des valeurs respectives de $8 \pm 4$ et $321 \pm$ 22. Sur l'ensemble de la période de suivi, il n'y a pas de différence significative au seuil de 5 p. 100 entre la DO moyenne obtenue avec les sérums des animaux infectés et celles des témoins négatifs (mouton $1: t=0,33$ pour ddl 219 , et, mouton $2: t=1,18$ pour ddl 139 ).

\section{Comparaison de la sensibilité du test de Woo et de I'ELISA T. vivax}

Sur 330 échantillons provenant d'animaux activement infectés, le test de Woo a fourni 54 p. 100 de résultats positifs tandis que l'ELISA n'en a diagnostiqué que $2,1 \mathrm{p}$. 100 (tableau I). Aucun outil mathématique n'est nécessaire pour montrer la supériorité du test de Woo pour la détection de l'infection.

\section{Test en aveugle}

Sur les 42 sérums sélectionnés pour le test en aveugle, 23 provenant du mouton 1 , et 19 provenant du mouton 2 ont été testés au CIRDES et à l'ILRI. Les DO obtenues dans ces laboratoires et celui de Guyane sont indiquées au tableau II (pour une bonne visualisation des résultats les DO supérieures au seuil de positivité fixé par l'ILRI, valeur 50 , figurent en caractère gras). Les jours de prélèvements et les parasitémies des animaux sont également indiqués (le prélèvement à Jo étant réalisé avant l'inoculation, le numéro correspond au nombre de jours après inoculation).

Pour ces séries appariées, en réalisant une analyse qualitative des résultats (test positif si DO $>49$ ), on n'observe pas de différence significative, au risque de 5 p. 100 , entre les résultats du laboratoire du CIRAD-EMVT-Guyane et de l'ILRI $(\mathrm{CHI} 2$ corrigé $=0$ avec ddl = 1), ni entre ceux du CIRAD-EMVT-Guyane et du CIRDES (CHI 2 corrigé $=1,5$ avec $d d l=1$ ). 
TABLEAU I

Prélèvements effectués et leurs résultats au test de Woo et à l'ELISA T. vivax

\begin{tabular}{|c|c|c|c|c|c|c|}
\hline \multirow{2}{*}{$\begin{array}{l}\text { Animal et période } \\
\text { de suivi }\end{array}$} & \multirow[t]{2}{*}{ Duree du suiv? } & \multirow{2}{*}{$\begin{array}{l}\text { Nombre de prélèvements } \\
\text { quotidiens }\end{array}$} & \multicolumn{2}{|c|}{$\begin{array}{l}\text { Positifs } \\
\text { au test de Woo }\end{array}$} & \multicolumn{2}{|c|}{$\begin{array}{l}\text { Positifs } \\
\text { au test ELISA } \\
\text { de T. Vivax }\end{array}$} \\
\hline & & & Nombre & p. 100 & Nombre & p. 100 \\
\hline Mouton 1 j periode 1 . & 130 ours & 113 & 85 & $75 \mathrm{p} \cdot 100$ & 4 & $3.5 \mathrm{p} .100$ \\
\hline $\begin{array}{l}\text { Mouton 1, période } 2 \\
\text { J131.j285 }\end{array}$ & 155 jours & 92 & 5 & $5,4 p, 100$ & 0 & 0.p. 100 \\
\hline Mouton 2: J1-J130 & 130 jours & 125 & 89 & $71 \mathrm{p} .100$ & 3 & 24 P. 100 \\
\hline Total & 415 jours & 330 & 179 & $54 \mathrm{p}, 100$ & 7 & 21 p. 100 \\
\hline
\end{tabular}

TABLEAU II

Jours de prélèvements, parasitémies et DO obtenues dans les trois laboratoires sur 42 échantillons testés en aveugle

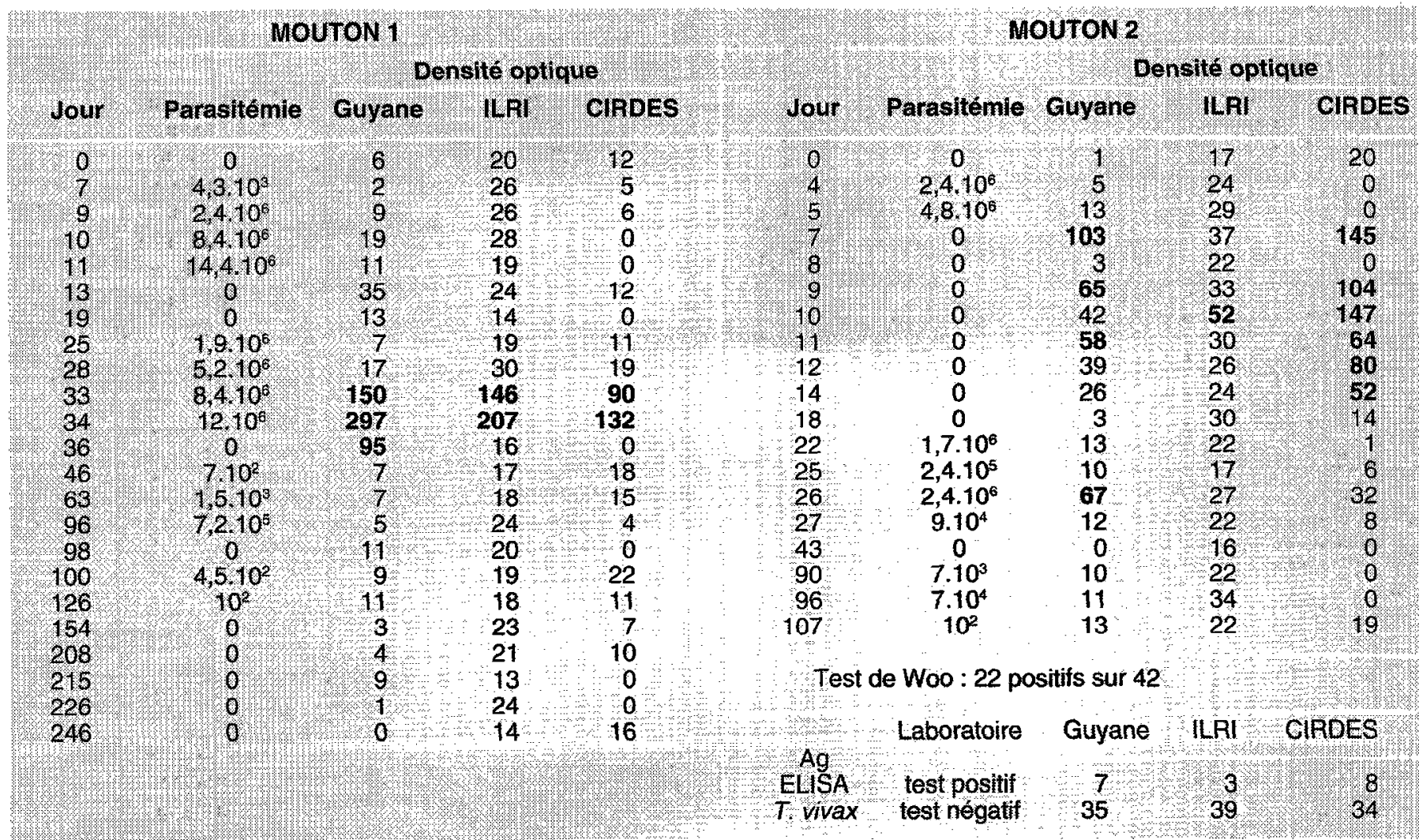


En réalisant une analyse quantitative, sur les 40 examens effectués sur des échantillons prélevés pendant la période d'infection, les DO moyennes sont pour le CIRDES : $26 \pm 13$; pour le CIRAD-EMVT-Guyane : $30 \pm 17$, et pour l'ILRI : $31 \pm 11$.

Les comparaisons de moyennes 2 à 2 des séries appariées de grand échantillons $(n>30)$ ne permettent pas de mettre en évidence de différence significative entre les DO moyennes obtenues dans les trois laboratoires, au risque de 5 p. 100 (écarts réduits : $\varepsilon$ ILRI/Guyane $=0,23$; $\varepsilon \mid \mathrm{LRI} / \mathrm{CIRDES}=0,7$, et $\varepsilon$ CIRDES/Guyane $=-0,6)$.

\section{DISCUSSION}

Les résultats comparés des laboratoires ne montrent pas de différence importante entre les diagnostics effectués à I'ILRI, au CIRDES ct au CIRAD-EMVT-Guyane. La nature des plaques, IMMULON B DYNATECHND ou NUNC MAXISORPND, ne modifie pas les résultats de manière significative. Ceci confirme les observations faites antérieurement par $\mathrm{G}$. Duvallet (communication personnelle).

Selon Nantulya et al. $(9,10,11,12)$ le test de détection des antigènes de $T$. vivax est plus sensible que le test de Woo. Les antigènes recherchés étant internes, le test pourrait rester négatif pendant les premières périodes de l'infection (10 à 20 jours), jusqu'à ce qu'une quantité importante d'antigènes soit libérée par la lyse des parasites. Par la suite le test resterait positif, même pendant les périodes aparasitémiques, et jusqu'à une quinzaine de jours après un traitement curatif.

Dans cette expérience il a effectivement été observé que cet ELISA peut rester négatif pendant les premiers jours d'infection, la détection des antigènes ne devenant positive qu'à J34 et J8. Par contre, la sensibilité du test s'est avérée très inférieure à celle du test de Woo.

Le résultat du test après traitement trypanocide lors d'une forte parasitémie n'a été positif que dans un cas sur quatre (mouton 2 après le premier traitement). II faut noter que la DO a légèrement progressé lors du premier traitement du mouton 1 , sans atteindre le seuil de positivité. Enfin dans les autres cas les traitements lors de fortes parasitémies n'ont eu aucun effet sur la valeur des DO post-traitement, bien qu'une forte libération d'antigènes ait eu lieu. La DO évoluant parfois en relation avec la parasitémie, parfois sans relation apparente, les pics d'antigénémie décrits par Nantulya et al. n'ont pas été observés. Enfin, il n'a pas été enregistré de résultats positifs pendant une longue période aparasitémique.

Devant la faible sensibilité du test de détection des antigènes, Kanwe et al. (6) ont proposé d'abaisser le seuil de positivité indiqué par l'ILRI (DO 50). Dans cette expérience, les DO moyennes des animaux infectés étant de 10,5 \pm 4 et $12 \pm 2$, pour enregistrer un taux de positivité satisfaisant, il faudrait abaisser le seuil de positivité jusqu'à des valeurs si basses (< 10 par exemple) qu'elles se situeraient en dessous du seuil de sensibilité des lecteurs ELISA. Enfin le nombre de faux positifs serait considérable.

L'IAEA a établi un nouveau protocole dans lequel les résultats sont fournis sous forme de pourcentage de positivité par rapport à un sérum standard.

$\mathrm{Au}$ vu des présents résultats, ces techniques sont sans appel, puisque les DO au cours de l'infection ont été souvent voisines de zéro, et ne sont pas différentes des DO pré-infection ou de celles des témoins négatifs.

Force est de constater que I'ELISA par immunocapture qui a été utilisé dans cette expérience n'est pas en mesure de fournir un diagnostic fiable de l'infection par la souche de T. vivax isolée en Guyane française.

L'existence, en Afrique, de souches présentant les mêmes caractéristiques que la souche de Guyane française est hautement probable puisque les parasites présents en Amérique du Sud sont d'origine africaine. Certains résultats récemment publiés l'indiquent, ainsi, Kanwe et al. (6), au Burkina Faso, et Faye (3), en Gambie, obtiennent respectivement 9,9 p. 100 et 11 p. 100 de résultats positifs avec l'ELISA par immunocapture d'antigènes de $T$. vivax sur des animaux trouvés infectés par $T$. vivax aux tests parasitologiques.

\section{CONCLUSION}

Les diagnostics réalisés dans trois laboratoires différents ont fourni des résultats sensiblement identiques.

Pour ces deux moutons expérimentalement infectés avec une souche guyanaise de $T$, vivax, la sensibilité du test de détection des antigènes par ELISA pendant les 130 et 285 premiers jours d'infection a été très basse (en moyenne 2,1 p. 100 de résultats positifs), de loin inférieure à celle de la technique de Woo (en moyenne 54 p. 100 de résultats positifs). L'association des deux techniques de diagnostic n'apporte aucune sensibilité supplémentaire.

Les DO enregistrées au cours de ces longues périodes d'infections expérimentales sont trop basses pour permettre de distinguer les animaux infectés des animaux non infectés. L'antigène reconnu par le monoclonal utilisé pour ce test est présent, mais faiblement disponible, probablement produit en quantité insuffisante et/ou de manière irrégulière par la souche parasitaire utilisée. Une formation importante d'immuncomplexes pourrait également expliquer ce phénomène. On ignore pour le moment les raisons de cette faible disponibilité, tant pour la souche de Guyane française que pour celles d'Afrique de l'Ouest.

La sensibilité insuffisante de ce test, observée également en Afrique, montre que des souches présentant des caractéristiques voisines, sont présentes sur ce continent, et risquent de fausser considérablement les résultats des enquêtes épidémiologiques entreprises à l'aide de ce kit. 
Le test de Woo ne présentant une sensibilité satisfaisante que pendant la première phase des infections, ce test de détection des antigènes par ELISA étant inutilisable, et la détection des anticorps ne permettant pas de savoir si la présence du parasite est contemporaine du prélèvement, on ne possède actuellement aucun outil de diagnostic satisfaisant pendant les phases aparasitémiques. Il serait intéressant d'éprouver la sensibilité des techniques de PCR au cours de cette phase. Quol qu'il en soit, pour des enquêtes épidémiologiques de grande envergure, les techniques de PCR n'étant pas adaptées, il est nécessaire de développer de nouveaux monoclonaux plus sensibles pour la détection des infections par T. vivax.

\section{Remerciements}

Nous remercions vivement $M$. $G$. Duvallet du CIRDES (ex-CRTA) à Bobo-Dioulasso, et M. S. Minja de l'ILRI (exILRAD), à Nairobi, pour la réalisation des tests ELISA en aveugle, ainsi que M. G. Uilenberg qui a accepté de relire notre manuscrit.

\section{Bibliographie}

1. BETANCOURT A.E., RAMIREZ L.E., WELLS E.A., BAZALAR H., 1979. La tecnica de centrifugacion en tubo capilar en el diagnostico de tripanosomiasis experimental. Revta ICA, Bogota (Colombia), 14 : 97-104.

2. DESQUESNES M., LA ROCQUE S. de, PEREGRINE A.S. French Guyanan stock of Trypanosoma vivax resistant to diminazene aceturate but sensitive to isometamidium chloride. Acta trop. (sous presse).

3. FAYE J., 1993. The use of an antigen-detection FI ISA for the diagno- sis of trypanosome infections in N'Dama cattle in Gambia. In : Improving the diagnosis and control of trypanosomiasis and other vector-borne diseases of African livestock using immunoassay methods, IAEATECDOC-707. Vienna, Austria, IAEA, p. 51-57.

4. FERENC S.A., STOPINSKI V., COURTNEY C.H., 1990. The develoment of an enzyme-linked immunosorbent assay for Trypanosoma vivax and its use in a seroepidemiological survey of the eastern Caribbean Basin. Int. J. Parasitol., $20: 51-56$

5. HOARE C.A., 1972. The trypanosomes of mammals. Edinburgh, U.K., Backwell Scicntific Publications ; Bristol, U.K., Western Printing Services Ltd, p. 125-141.

6. KANWE A.B., BENGALY Z., SAULNIER D., DUVALLET G., 1992. Evaluation du test de détection des antigènes circulants de trypanosomes à l'aide d'anticorps monoclonaux. Infections expérimentales et naturelles. Revue Élev. Méd. vét. Pays trop., 45 : 265-271.

7. LANCELOT R., 1988. La trypanosome bovine à Trypanosoma vivax en Guyane française, contribution à l'étude clinique et épidémiologique. Thèse de Doctorat vétérinaire, ENVA, Maisons-Alfort, France.

8. MURRAY M., MURRAY P.K., McINTYRE W.I.M., 1977. An improved parasitological technique for the diagnosis of African trypanosomiasis. Trans. R. Soc. trop. Med. Hyg., 71 : 325-326.

9. NANTULYA V.M., 1990. Trypanosomiasis in domestic animals: the problem of diagnosis.Rev. sci. tech. Off. int. Epiz., 9:357-367.

10. NANTULYA V.M., 1991. Molccular diagnosis of parasites. Experientia, $47: 142-145$

11. NANTULYA V.M., LINDQVIST K.J., 1989. Antigen-detection enzyme immunoassays for the diagnosis of Trypanosoma vivax, $T$. congolense and T. brucei infections in cattle. Trop. Med. Parasit., 40:267-272.

12. NANTULYA V.M., MUSOKE A.J., RURANGIRWA F.R., SAIGAR N., MINJA S.H., 1987. Monoclonal antibodies that distinguish Trypanosoma congolense, T. vivax and T. brucei. Parasite Immunol, 9 : 421-431.

13. SCHWARTZ D., 1963. Méthodes statistiques à l'usage des médecins et des biologistes. Paris, France, Flammarion Médecine-Sciences, p. 303.

14 WOO P.T.K., 1970. The haematocrit centrifuge technique for the diagnosis of African trypanosomiasis. Acta trop., $27: 384-386$
DESQUENES (M.), LA ROCQUE (S. de). Comparison of the sensitivity of Woo's test and a test for detecting antigens of Trypanosoma vivax in two sheep experimentally infected with a Guyanese strain of the parasite. Revue Élev. Méd. vét. Pays trop., 1995, 48 (3) : 247-253.

A trapping ELISA for the detection of circulating antigens of Trypanosoma vivax with a specific monoclonal antibody was developed by Nantulya and Lindqvist in 1989. With the African strains tested, its sensitivity proved very high. Using reagents supplied by ILRI, the sensitivity of this test was compared with that of the Woo test in two sheep experimentally infected with a Guyanese strain of $T$. vivax. Blind tests carried out at CIRDES and ILRI confirmed the results obtained in French Guiana. The animals were bled regularly during the first 130 and 285 days of infection. Whatever the period of infection ans whether there was patent parasitaemia or not, the mean sensitivity of the ELISA test was very low $: 2.1 \%$ of positive results, far below the Woo test's $54 \%$ of positive results. Associating the two techniques did not improve the sensitivity. The mean optical densities recorded during these long periods of infection $(0.010 \pm 0.004$ and $0.012 \pm 0.002)$ are so close to the sensitivity threshold of the ELISA readers that they cannot be proposed as a cut-off threshold to improve the sensitivity of the test. A complementary study of the sensitivity of this test is in progress at ILRI.

As already observed in Burkina Faso and The Gambia, this poor sensitivity may greatly affect the results of the epidemiological surveys being carried out with these reagents in Africa. New monoclonal antibodies must be developed to produce a test for $T$. vivax antigens with more satisfactory sensitivity.

Kew words : Sheep - Trypanosoma vivax - Experimental infection ELISA - Antigen - Antibody - Serology - French Guiana.
DESQUESNES (M.), LA ROCQUE (S. de). Estudio comparativo de la sensibilidad del test de Woo y de un test de detección de antígenos de Trypanosoma vivax en dos corderos infectados en forma experimental con una cepa guayanesa del parásito. Revue Élev. Méd. vét. Pays trop., $1995,48(3): 247-253$

En 1989, Nantulya y Lindqvist establecieron un test de ELISA para la detección de antígenos circulantes de Trypanosoma vivax, por inmunocaptura, gracias a un anticuerpo monoclonal específico. La sensibilidad de las cepas africanas fue muy elevada. Gracias a reactivos facilitados por el ILRI, la sensibilidad fue comparada a la del test de Woo, gracias a infecciones experimentales en dos corderos con una cepa guayanesa de $T$. vivax. Mediante tests ciegos, realizados en el CIRDES y el ILRI, se logró confirmar los resultados obtenidos en la Guayana Francesa. Se obtuvieron regularmente muestras de los animales durante los 130 y $\mathbf{2 8 5}$ primeros días de la infección. En todo momento de la infección, con detección de parasitemia o no, la sensibilidad del ELISA fue muy baja : en promedio 2,1 p. 100 de resultados positiyos, muy inferior à la de la técnica de Woo : $54 \mathrm{p}$. 100 de resultados positivos. La asociación de las dos técnicas no aporta ninguna sensibilidad suplementaria. Las densidades ópticas promedio, registradas durante estos dos largos períodos de infección, $0,01 \pm 0,004$ y $0,012 \pm 0,002$, son tan cercanas al umbral de sensibilidad de los lectores ELISA, que no pueden proponerse como límite para mejorar la sensibilidad de positividad del test. Un estudio complementario de la sensibilidad de este test está en curso en el ILRI. Este defecto de sensibilidad, ya observado en Burkina Faso y Gambia, podría alterar fuertemente los resultados de las encuestas epidemiológicas ilevadas a cabo con los reactivos en Africa. Con el fin de lograr un test de detección de antígenos de $T$. vivax con une sensibilidad satisfactoria, deben de desarrollarse nuevos anticuerpos monoclonales.

Palabras clave : Ovino - Trypanosoma vivax - Infección experimental ELISA - Antígeno - Anticuerpo - Serología - Guayana Francesa 